\title{
Performance enhancement for a non-orthogonal multiple access system using $4 \times 4$ multiple-input multiple-output visible-light communication
}

\author{
Ibrahim A. Elewah $\odot$, ${ }^{\text {a,b }}$ Faezah Jasman $\odot,{ }^{a}$ and Shashiong $\mathbf{N g} \oplus^{\text {a,* }}$ \\ ${ }^{a}$ Universiti Sains Malaysia, Institute of Nano Optoelectronics Research and Technology, \\ Penang, Malaysia \\ ${ }^{\mathrm{b}}$ American College of the Middle East, Department of Engineering and Technology, Kuwait
}

\begin{abstract}
A multi-user visible-light communication (VLC) system based on $4 \times 4$ multipleinput multiple-output (MIMO) was investigated. The system consists of four light-emitting diodes (as a transmitter) and four photodetectors (as a receiver). The proposed system was simulated for two, three, and four users. The objective of this study is to optimize the system power consumption and to utilize the sum rate on the system coverage area. By expanding the system from a $2 \times 2$ MIMO-VLC to a $4 \times 4$ MIMO-VLC, the results demonstrate that the total sum rate of the system can be improved. The findings reveal that the $4 \times 4$ MIMO-VLC achieves a maximum sum-rate enhancement of $143 \%$ compared with the $2 \times 2$ MIMO-VLC. The results also show that the efficiency of $4 \times 4$ MIMO-VLC system with non-orthogonal multiple access (NOMA) and gain ratio power allocation (GRPA) was increased by around $181 \%$ compared to $2 \times 2$ MIMO-NOMA-based VLC with GRPA in combination. For the $4 \times 4$ MIMONOMA-VLC device with GRPA, the sum bit rate for two, three, and four users was increased by $40 \%, 33 \%$, and $24 \%$, respectively. Through this study, it can be concluded that GRPA scheme plays an important role in utilizing MIMO-VLC system sum rate of the MIMO-VLC system. () The Authors. Published by SPIE under a Creative Commons Attribution 4.0 Unported License. Distribution or reproduction of this work in whole or in part requires full attribution of the original publication, including its DOI. [DOI: 10.1117/1.OE.59.12.126104]
\end{abstract}

Keywords: visible-light communication; multiple-input multiple-output; non-orthogonal multiple access; gain ratio power allocation.

Paper 20200997 received Aug. 22, 2020; accepted for publication Nov. 16, 2020; published online Dec. 4, 2020.

\section{Introduction}

Although ease of use and mobility are two important features provided by wireless devices, research interests to find other alternatives have been emerging in the field of optical wireless communications, especially visible-light communication (VLC) technology. ${ }^{1}$ This is because VLC offers various advantages, such as license-free wide bandwidth, no electromagnetic interference, and low-power consumption. ${ }^{2-4}$ Moreover, VLC transmitters use high-brightness lightemitting diodes (LEDs) rather than the incandescent and fluorescent lamps of traditional light transmitters. Unique features of LEDs include higher lighting efficiency, cost-effectiveness, longer lifetimes, smaller size, and easier maintenance. ${ }^{5}$ Apart from that, a new heterogeneous radio frequency (RF)/VLC industrial network architecture is developed to support uplink and downlink communication services. ${ }^{6}$

Due to its outperformance of other technologies, VLC has recently attracted the attention of researchers, particularly in Europe. Spectral efficiency and LED exploring capabilities were enhanced using a non-return-to-zero on-off keying modulation scheme, where the data rate reached was 100 Mbps. $^{7}$ In a single-input single-output architecture, a data rate of $3.5 \mathrm{Gbps}$ was recently achieved using a single-color micro-LED. ${ }^{8}$

To date, a range of schemes has been introduced to boost efficiency and expand the VLC system coverage. ${ }^{9}$ The most common and efficient method is the multiple-input multiple-output

*Address all correspondence to Shashiong Ng, shashiong@usm.my 
(MIMO) scheme, with arrays of illuminating LEDs. ${ }^{10,11}$ Meanwhile, 150 Mbps over $6 \mathrm{~m}$ using on-off keying and $100 \mathrm{Mbps}$ over $0.6 \mathrm{~m}$ using orthogonal frequency-division multiplexing (OFDM) modulation with a $2 \times 2$ MIMO was demonstrated in Refs. 11 and 12. Also, an MIMO-OFDM-based VLC system with four separated LEDs and a $3 \times 3$ receiver array as a detector transmitting with 1 Gbps was reported. ${ }^{12}$

The key factor used to distinguish between various types of electromagnetic and optical wireless systems is the multiple access schemes. There are two distinct approaches to multiple access systems, namely, the orthogonal multiple access approaches and the non-orthogonal multiple access (NOMA). A receiver in an orthogonal scheme uses functions to separate undesired signals from desired ones. This means that in orthogonal schemes, different users' signals are orthogonal to one another. ${ }^{13}$

Over the past few years, researchers have shown more interest in NOMA schemes due to their superior spectral efficiency. As a result, they have been proposed for fifth-generation mobile communications via power domain multiplexing. ${ }^{14}$ In addition, NOMA allows all users to employ the system modulation bandwidth. This is accomplished using power domain overlay coding on each transmitter ${ }^{15}$ and by applying successive interference cancellation (SIC) on each receiver $^{16}$ as well. It has been suggested that implementing NOMA on MIMO-VLC system achieves a significant improvement in the signal-to-noise ratio for various configurations. ${ }^{17,18}$ Apart from the NOMA approach, physical devices such as precoder and equalizer were also used to mitigate different types of interference. ${ }^{19}$ Some other researches ${ }^{20}$ have introduced a new receiver structure employing selective combining technique to reduce background noise effect.

Many MIMO-NOMA-based VLC systems have been experimentally tested, yet without applying dynamic power allocation schemes. Numerous studies showed significant enhancement in system performance compared with statistic power allocation. ${ }^{21}$ Moreover, several methods for power allocation in MIMO-NOMA RF systems have been proposed, such as hybrid precoding ${ }^{22,23}$ and signal adjacency. ${ }^{24}$ Nevertheless, one of the major drawbacks of those methods is their high processing complexity. In the broad implementation of the MIMO-NOMA mechanism, effective power allocation methods such as gain ratio power allocation (GRPA) and normalized gain difference power allocation with low processing complexity are needed for practical VLC systems. ${ }^{25,26}$

In this study, a $4 \times 4$ MIMO-NOMA-VLC system was proposed to improve the sum rate and to optimize the power consumption of the system. The GRPA was introduced to realize an effective and less complex power allocation scheme. The overall performance of the system was measured through numerical simulations. Compared to a $2 \times 2$ MIMO-NOMA-VLC system, results show a significant improvement in the sum rate of an indoor $4 \times 4$ MIMO-NOMAVLC system with or without the GRPA method. The results show that after applying GRPA on $4 \times 4$ MIMO-NOMA-VLC, the achievable sum rate was improved. The achievable sum rates for 2,3 , and 4 users were $96 \%, 98 \%$, and $99 \%$ of the maximum achievable sum rate (at the center of the system), respectively.

\section{System Model}

This section presents the mathematical model of the proposed MIMO-NOMA-based multi-user VLC device. Figure 1 shows the $4 \times 4$ MIMO-NOMA-VLC device with $K$ users. Each of the users has four photodiodes (PDs) and have access to the bandwidth of all four LEDs. Additionally, this proposed MIMO-NOMA-VLC system is compatible with the DC-biased optical OFDM (DCO-OFDM) modulation. ${ }^{27}$.

Figure 2 shows a $4 \times 4$ MIMO-NOMA-VLC system block diagram with DCO-OFDM modulation. Consider $x_{i}(t)$ to be the input signals of $\operatorname{LED} i(i=1,2,3$, or 4$)$. The acquired $i$ 'th LED input signal can be analyzed using

$$
x_{i}(t)=\sum_{k}^{K} \sqrt{\rho_{i, k}} S_{i, k}(t)+I_{\mathrm{DC}}
$$




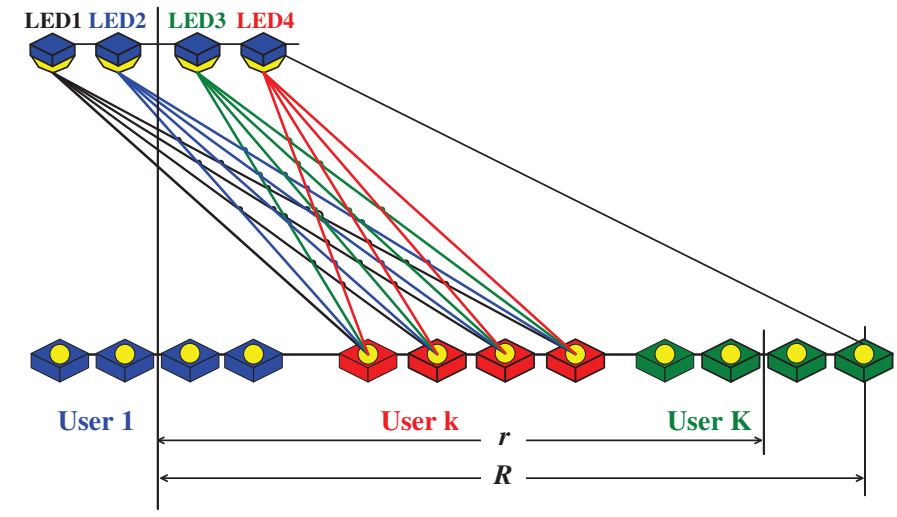

Fig. 1 Illustration of a $4 \times 4$ MIMO-NOMA-based VLC system with $K$ users.

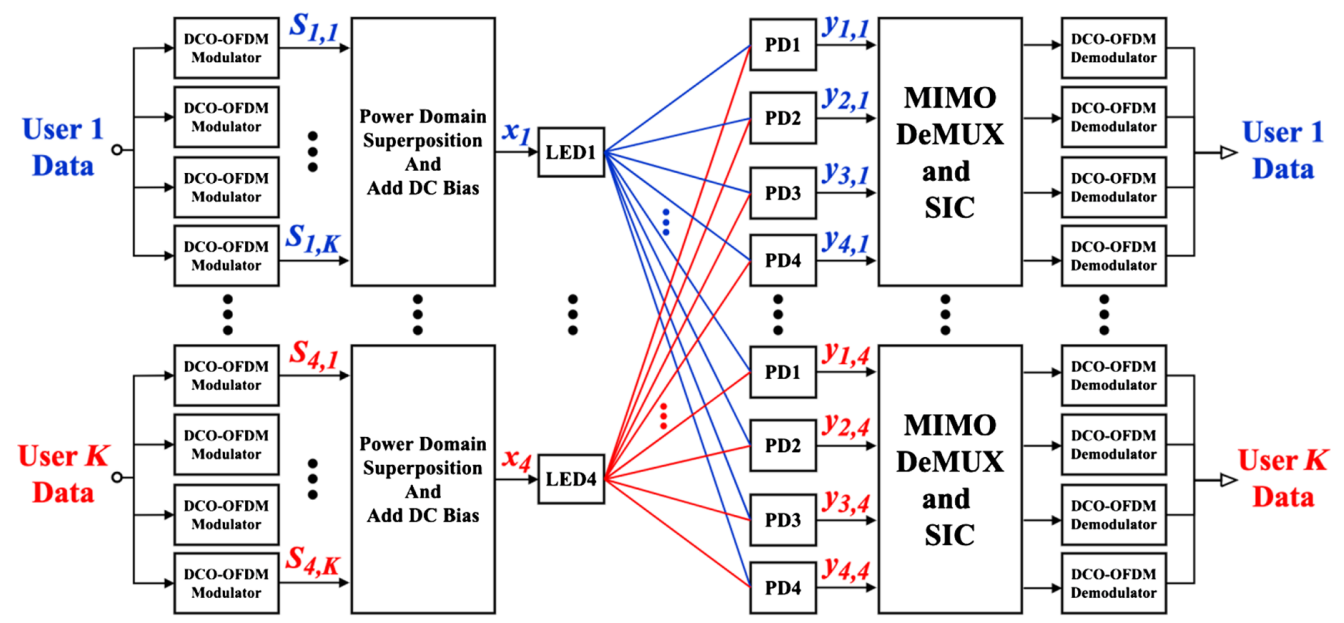

(a)

(b)

Fig. $24 \times 4$ MIMO-NOMA-based VLC block diagram with $K$ users. (a) $2 \times 2$ MIMO-NOMA based multi-user VLC system. (b) $4 \times 4$ MIMO-NOMA based multi-user VLC system.

where $S_{i, k}(t)$ represents the signal prepared for the $k$ 'th user $(k=1,2, \ldots, K)$ in the $i$ 'th LED. LED electrical power is the electrical power consumed by the LED to produce corresponding optical power. The electrical power allocated in the specified LED for the specified user is $\rho_{i, k}$, and the DC bias for every LED is $I_{\mathrm{DC}}$. Each LED should have constant overall electrical power, and the following electrical power constraint is used:

$$
\sum_{k=1}^{K} \rho_{i, k}=P_{\text {elec. }}
$$

It is assumed that each LED is stimulated with enough electrical power to, $P_{\text {elec. }}$ so the generality will not be lost and the produced average transmitted optical power, $P_{\text {opt }}=10 \mathrm{~W}$. After the transmission, the vector of the electrical signal received by the $k$ 'th user can be written as ${ }^{28}$

$$
y_{k}=\gamma P_{\mathrm{opt}} \xi H_{k} x+n_{k},
$$

where $\gamma$ is the responsivity of the PD, the modulation index is, $\xi$ and $H_{k}$ is the four-channel matrix of the $k$-users. $x=\left[\begin{array}{llll}x_{1} & x_{2} & x_{3} & x_{4}\end{array}\right]^{T}$ is the vector of the transmitted electrical signal, and $n_{k}$ is the additive noise vector. Assuming that a non-imaging receiver (NimR) is used, $H_{\text {NImR }}$ represents the channel matrix for the $4 \times 4$ MIMO-VLC system employing a NimR and is given as 


$$
H_{\mathrm{NImR}}=\left[\begin{array}{llll}
h_{11} & h_{12} & h_{13} & h_{14} \\
h_{21} & h_{22} & h_{23} & h_{24} \\
h_{31} & h_{32} & h_{33} & h_{34} \\
h_{41} & h_{42} & h_{43} & h_{44}
\end{array}\right],
$$

where $h_{j i}(i, j=1,2,3,4)$ is the optical channel gain between the $j$ 'th PD and the $i$ 'th LED. This Eq. (4) has been extended to $4 \times 4$ and as it was used for $2 \times 2$ MIMO-VLC in Ref. 27 .

In the $4 \times 4$ MIMO-VLC system, there are sixteen possible vectors to be evaluated between the LEDs and PDs, as shown in Fig. 1. As compared with the four vectors in case of $2 \times 2$ system, these sixteen vectors would expand the mathematical model, and hence increase the complexity of the system. In addition, this would increase the inter-cell-interference (ICI) effect. To minimize the ICI effect and to enhance the performance of the system, NOMA with GRPA approach was applied.

In a typical room situation with VLC, several LEDs are fixed on the ceiling of the room. While each PD is capable of detecting components of both non-line of sight components (NLOS) and line of sight (LOS). In the present work, only the LOS components were taken into consideration because it provides much higher intensity compared to that of the NLOS components. ${ }^{29}$ The generalized Lambertian radiation pattern model is applied to find the LED's LOS irradiance. The LOS optical channel gain, $h_{j i, k}$ of the $k^{\prime}$ th user between the $i$ 'th $\left(i=1,2,3\right.$, and 4) LED, and the $j^{\prime}$ th $\left(j=1,2,3\right.$, and 4) PD is calculated as ${ }^{30}$

$$
h_{j i}=\frac{(m+1) A}{2 \pi d_{j i}^{2}} \mu \eta \cos ^{m}\left(\varphi_{j i}\right) \cos \left(\phi_{j i}\right),
$$

where

$$
m=\frac{-\ln 2}{\ln \cos \left(\psi_{1 / 2}\right)} .
$$

Here $\psi_{1 / 2}$ is the LED semi-angle at half power, $\mu$ is the optical filter gain, $\eta$ is the gain of the optical lens, $A$ is the PD active area, $d_{j i}$ is the distance between $i$ 'th LED and the $j$ 'th PD, $\varphi$ is the angle of irradiance, and $\phi$ is the angle of incidence. The optical gain will be zero if $\phi$ is outranged of the receiver field of view. De-multiplexing MIMO is applied with zero-forcing (ZF) to recover the sent data with basic channel inversion because of its simplicity. ${ }^{27}$ After applying the ZF de-multiplexing, at the $k^{\prime}$ th user, the expected vector of the electrical signal is evaluated using

$$
\tilde{x}_{k}=x+\frac{1}{\gamma P_{\mathrm{opt}} \xi} H_{k}^{-1} n_{k}
$$

where $H_{k}^{-1}$ is the inverse of $H_{k}$. Consequently, SIC is applied to all LEDs for all users. To apply SIC, the decoding order of the users must be calculated for every LED. Sorting the users is done by finding the optical channel's sum of every user for every LED. ${ }^{21}$ Without a loss of generality, it is assumed that $K$ users for the $i$ 'th LED are sorted in descending order of their sum optical channel gains as follows:

$$
\begin{gathered}
h_{1 i, 1}+h_{2 i, 1}+h_{3 i, 1}+h_{4 i, 1}>h_{1 i, 2}+h_{2 i, 2}+h_{3 i, 2}+ \\
h_{4 i, 2}>\ldots>h_{1 i, K}+h_{2 i, K}+h_{3 i, K}+h_{4 i, K} .
\end{gathered}
$$

With reference to the $i$ 'th LED, the decoding order is then set to be

$$
O_{i, 1}<O_{i, 2}<\ldots<O_{i, K} .
$$

A detailed mathematical model of SIC can be found in Ref. 21. Finally, the data for all $K$ users are successfully extracted using the DCO-OFDM demodulation mechanism. 
Next step is to evaluate the sum rate of each case (for $k=2,3$, and 4 users). Equation (10) is used to calculate the sum rate for $4 \times 4$ MIMO-VLC system.

$$
\text { Sum Rate }=\operatorname{Re}\left(\sum_{k=1}^{K} \sum_{i=1}^{4} \frac{\frac{B W}{2} \log _{2}\left(1+S N R_{i k}\right)}{10^{6}}\right)
$$

where $\mathrm{BW}$ is the transmission bandwidth, $S N R_{i k}$ is the signal to noise ratio for each link between $i$ 'th LED and $k$ 'th user. Re operator is to evaluate the real part. It means equation's output will contain only real numbers (without imaginary numbers). The mathematical model of how to calculate the signal to noise ratio after adding the additive white Gaussian noise can be found in Ref. 27.

\section{Gain Ratio Power Allocation}

Power allocations schemes for optimizing NOMA are divided into two approaches: numerical search and strategic design. ${ }^{31}$. The low-power strategy is one of the recent strategic design methods and fractional transmit power allocations, ${ }^{32}$ where the allotted power is reduced as a channel gain ratio. In the NOMA power domain, a GRPA scheme, which considers user channel situations to ensure equal and efficient allocation, can boost the overall device performance. ${ }^{21}$

Due to its simplicity and effectiveness, GRPA has been proposed as a power allocation method for many NOMA-VLC systems. ${ }^{21}$ In the GRPA scheme, optical power relies on any optical channel of the device. Based on previous use of these systems, a generalized type of GRPA can be achieved by swapping the sum optical channel gain with the optical channel gain for MIMO-NOMA. As presented in Ref. 21, the relationship between the electrical powers allocated to user $k$ and user $k+1$ in accordance with to the $i$ 'th LED is given as

$$
\rho_{i, k}=\left(\frac{h_{1 i, k+1}+h_{2 i, k+1}+h_{3 i, k+1}+h_{4 i, k+1}}{h_{1 i, 1}+h_{2 i, 1}+h_{3 i, 1}+h_{4 i, 1}}\right)^{k+1} \rho_{i, k+1} \text {. }
$$

\section{Simulation Results}

In this section, the performance of the $4 \times 4$ MIMO-NOMA-VLC system with and without using the GRPA process was discussed. The $4 \times 4$ MIMO-NOMA-VLC system is simulated for two, three, and four users. In each case, at least one user is fixed and the other user(s) is/are moving from the system center to the system edge. Table 1 displays parameters and their value used for the simulation.

Table 1 System parameters and their values.

\begin{tabular}{llcc}
\hline \hline Symbol & \multicolumn{1}{c}{ Description } & Value (s) & Unit \\
\hline$P_{\mathrm{opt}}$ & Average optical power & 10 & Watt \\
$R$ & Radius of system coverage & 2 & $\mathrm{~m}$ \\
$\psi_{1 / 2}$ & LED semi-angle at half power & 60 & \\
$\mu$ & Optical filter gain & 0.9 & $\mathrm{~N} / \mathrm{A}$ \\
$\eta$ & Optical lens gain & 2.5 & $\mathrm{~N} / \mathrm{A}$ \\
BW & Band width & 10 & $\mathrm{MHz}$ \\
$\gamma$ & PDs responsivity & 0.53 & $\mathrm{~A} / \mathrm{W}$ \\
$\xi$ & Modulation index & 0.5 & $\mathrm{~N} / \mathrm{A}$ \\
\hline \hline
\end{tabular}


Elewah, Jasman, and Ng: Performance enhancement for a non-orthogonal multiple access system...

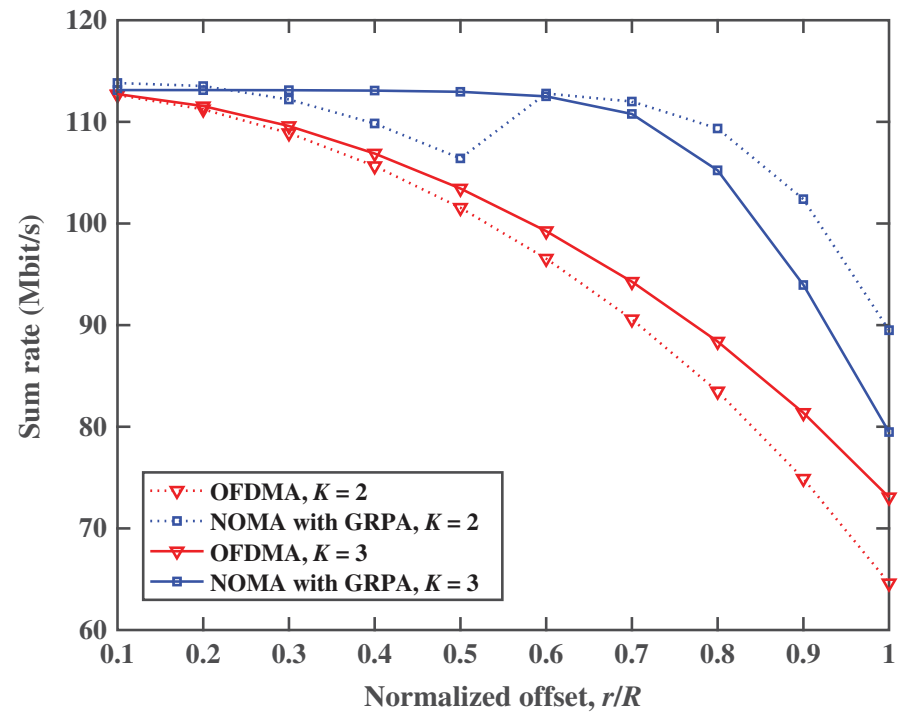

(a)

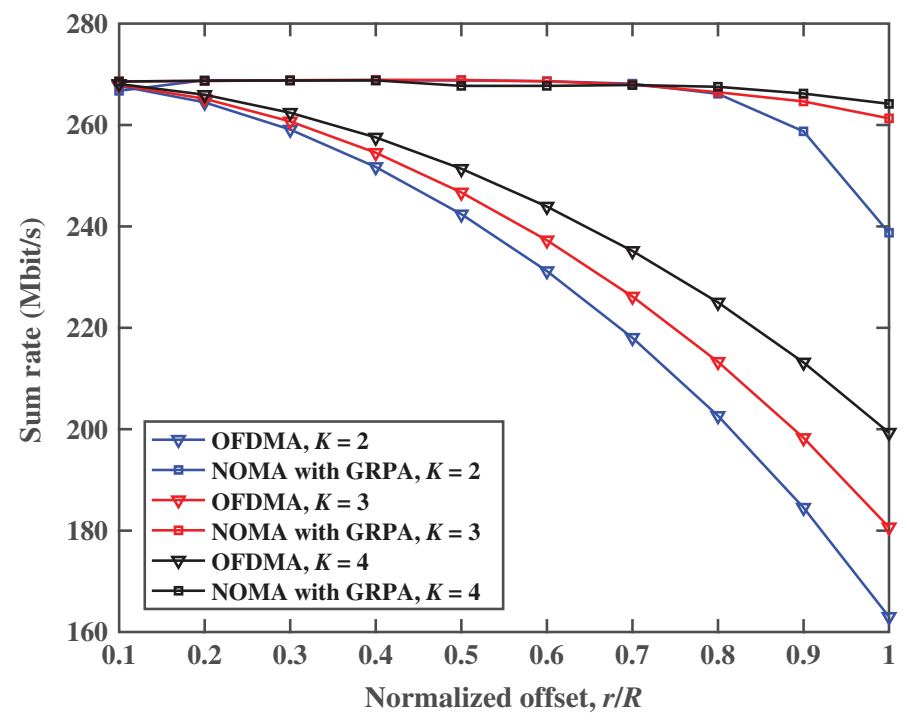

(b)

Fig. 3 Achievable sum rate versus normalized offset.

Figure 3(a) shows achieved sum rates of $2 \times 2$ MIMO-VLC using orthogonal frequencydivision multiple access (OFDMA) and NOMA with GRPA. While the achieved sum rates versus $r / R$ of $4 \times 4$ MIMO-VLC process using OFDMA and NOMA with GRPA are shown in Fig. 3(b).

Figure 4 shows the GRPA sum-rate gain over OFDMA versus $r / R$. The findings are summarized in Tables 2 and 3. The sum rate utilizing OFDMA decreases as the ratio $r / R$ rises in both $2 \times 2$ and $4 \times 4$ MIMO-VLC systems.

\subsection{Sum Rate Versus Normalized Offset}

In this section, the discussion is divided into three parts. The first section (Sec. 4.1.1) shows the system capacity enhancement after expanding a $2 \times 2$ (as reported in Ref. 26) to a $4 \times 4$ MIMOVLC system. For a detailed discussion, Figures 3(a) and 3(b) are used along with the first part of Table 2. The second part (Sec. 4.1.2) reveals the comparison between $2 \times 2$ and $4 \times 4$ MIMONOMA-VLC systems with applying GRPA at $K=3$. Figures 3(a) and 3(b), and the second part 
Elewah, Jasman, and $\mathrm{Ng}$ : Performance enhancement for a non-orthogonal multiple access system...

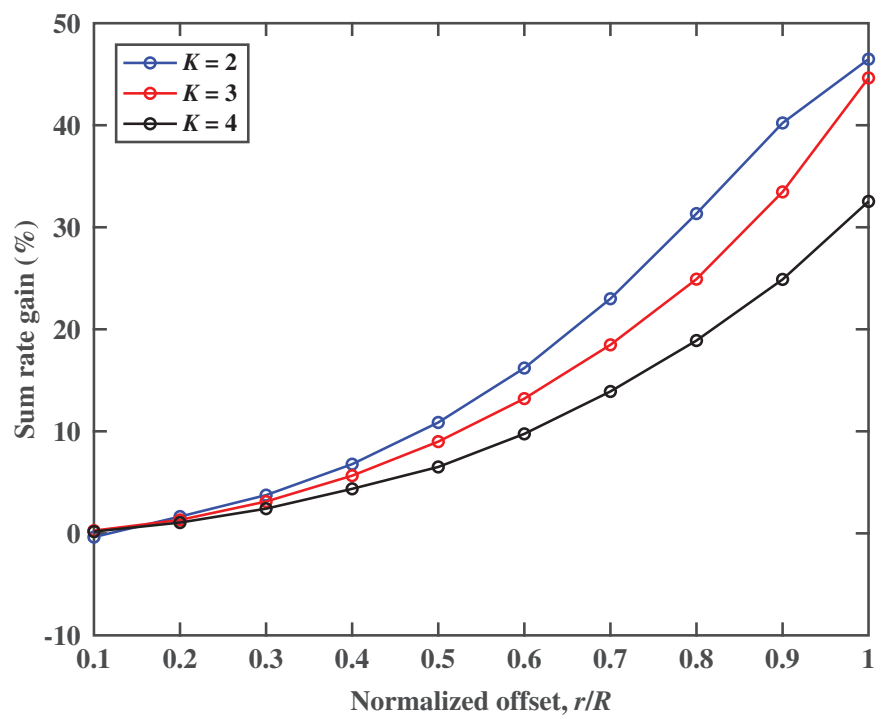

Fig. 4 Sum-rate gain of GRPA over OFDMA versus normalized offset.

Table $22 \times 2$ versus $4 \times 4$ MIMO-OFDMA and $2 \times 2$ versus $4 \times 4$ MIMO-NOMA-VLC with GRPA at $K=3$.

\begin{tabular}{|c|c|c|c|c|c|c|}
\hline \multirow[b]{3}{*}{$r / R$} & \multicolumn{3}{|c|}{$2 \times 2$ versus $4 \times 4$ MIMO-OFDMA } & \multicolumn{3}{|c|}{$2 \times 2$ versus $4 \times 4$ MIMO-NOMA-VLC with GRPA } \\
\hline & \multicolumn{2}{|c|}{ Sum rate $(\mathrm{Mbit} / \mathrm{s})$} & \multirow[b]{2}{*}{ Percentage (\%) } & \multicolumn{2}{|c|}{ Sum rate (Mbit/s) } & \multirow[b]{2}{*}{ Percentage (\%) } \\
\hline & $2 \times 2^{26}$ & $4 \times 4$ & & $2 \times 2$ GRPA $^{26}$ & $4 \times 4$ GRPA & \\
\hline 0.1 & 112.69 & 267.89 & 137.72 & 112.69 & 268.55 & 138.31 \\
\hline 0.2 & 111.54 & 265.18 & 137.74 & 112.89 & 268.73 & 138.05 \\
\hline 0.3 & 109.59 & 260.70 & 137.89 & 112.98 & 268.77 & 137.89 \\
\hline 0.4 & 106.88 & 254.51 & 138.13 & 112.99 & 268.78 & 137.88 \\
\hline 0.5 & 103.44 & 246.69 & 138.49 & 112.98 & 268.87 & 137.98 \\
\hline 0.6 & 99.25 & 237.26 & 139.05 & 112.51 & 268.60 & 138.73 \\
\hline 0.7 & 94.27 & 226.18 & 139.93 & 110.77 & 267.87 & 141.83 \\
\hline 0.8 & 88.39 & 213.31 & 141.33 & 105.23 & 266.45 & 153.21 \\
\hline 0.9 & 81.37 & 198.28 & 143.68 & 93.95 & 264.64 & 181.68 \\
\hline
\end{tabular}

of Table 2, are used in the analysis. Finally, the overall performance acquired from the $4 \times 4$ MIMO-VLC systems before and after applying GRPA for 2, 3, and 4 users is compared and presented in the third part (Sec. 4.1.3). Figure 3(b) is discussed in this section along with Table 3 to show the detailed values of each curve. The percentage columns in Tables 2 and 3 represents the percentage of second column out of first column of each part.

\subsection{1 $2 \times 2$ versus $4 \times 4$ MIMO-OFDMA}

The first part of Table 2 shows that the maximum sum rate achieved in the $2 \times 2$ MIMO-VLC system was $112.69 \mathrm{Mbit} / \mathrm{s}$ at $r / R=0.1$. After expanding the architecture to the $4 \times 4 \mathrm{MIMO}$ VLC system, the sum rate was enhanced to $137.72 \%$ (267.89 Mbit/s). Moreover, the enhancement percentage is increased with a higher normalized offset. Thus, system efficiency is 
Table $34 \times 4$ MIMO-VLC systems sum rate after and before applying GRPA at $K=2,3$, and 4 .

\begin{tabular}{|c|c|c|c|c|c|c|c|c|c|}
\hline \multirow[b]{3}{*}{$r / R$} & \multicolumn{3}{|c|}{$K=2$} & \multicolumn{3}{|c|}{$K=3$} & \multicolumn{3}{|c|}{$K=4$} \\
\hline & \multicolumn{2}{|c|}{ Sum rate (Mbit/s) } & \multirow[b]{2}{*}{$\begin{array}{l}\text { Percentage } \\
(\%)\end{array}$} & \multicolumn{2}{|c|}{ Sum rate (Mbit/s) } & \multirow[b]{2}{*}{$\begin{array}{c}\text { Percentage } \\
(\%)\end{array}$} & \multicolumn{2}{|c|}{ Sum rate (Mbit/s) } & \multirow[b]{2}{*}{$\begin{array}{c}\text { Percentage } \\
(\%)\end{array}$} \\
\hline & $4 \times 4$ & $\begin{array}{c}4 \times 4 \\
\text { GRPA }\end{array}$ & & $4 \times 4$ & $\begin{array}{c}4 \times 4 \\
\text { GRPA }\end{array}$ & & $4 \times 4$ & $\begin{array}{c}4 \times 4 \\
\text { GRPA }\end{array}$ & \\
\hline 0.1 & 268.55 & 268.55 & 00.00 & 268.55 & 268.55 & 00.00 & 268.55 & 268.55 & 00.00 \\
\hline 0.2 & 264.46 & 268.73 & 01.61 & 265.18 & 268.73 & 01.34 & 265.91 & 268.73 & 01.06 \\
\hline 0.3 & 259.10 & 268.77 & 03.73 & 260.70 & 268.77 & 03.10 & 262.42 & 268.77 & 02.42 \\
\hline 0.4 & 251.72 & 268.78 & 06.78 & 254.51 & 268.78 & 05.61 & 257.54 & 268.78 & 04.36 \\
\hline 0.5 & 242.40 & 268.87 & 10.92 & 246.69 & 268.87 & 08.99 & 251.37 & 267.71 & 06.50 \\
\hline 0.6 & 231.19 & 267.71 & 15.80 & 237.26 & 268.60 & 13.21 & 243.92 & 267.71 & 09.75 \\
\hline 0.7 & 218.08 & 267.87 & 22.83 & 226.18 & 267.87 & 18.43 & 235.17 & 267.87 & 13.90 \\
\hline 0.8 & 202.67 & 266.14 & 31.32 & 213.31 & 266.45 & 24.91 & 225.01 & 267.53 & 18.90 \\
\hline 0.9 & 184.53 & 258.74 & 40.22 & 198.28 & 264.64 & 33.47 & 213.16 & 266.20 & 24.88 \\
\hline
\end{tabular}

enhanced in the room borders or for the farthest user. The transmitted signals in the $4 \times 4$ MIMOVLC have 16 different routes (there are only four in the case of a $2 \times 2$ MIMO-VLC) to reach the corresponding user, which explains why the sum rate was enhanced over $100 \%$. In addition, the sum rate for $2 \times 2$ MIMO-VLC fluctuates at $K=2$ with the increase of normalized offset and a relatively low sum rate of about $106.4 \mathrm{Mbit} / \mathrm{s}$ is achieved at 0.5 normalized offset. This is due to the rate reduction of one of the LEDs at $r / R=0.5$, as reported in Ref. 26. From Fig. 3(b), it can be found that the proposed $4 \times 4$ MIMO-VLC does not suffer any fluctuation. In addition to that, the $4 \times 4$ MIMO-VLC system is preferred over $2 \times 2$ MIMO-VLC as it can maintain the same sum rate if one of the LEDs is malfunction.

\subsection{2 $2 \times 2$ versus $4 \times 4$ MIMO-NOMA-VLC with GRPA at $K=3$}

In this section, the sum rates acquired from $2 \times 2$ and $4 \times 4$ MIMO-NOMA-VLC systems with GRPA at $K=3$ is compared. From Fig. 3(b), it can be seen that the sum rate of the $4 \times 4$ MIMONOMA based VLC system with GRPA was increased for both $K=2$ and 3 as compared to that of the $2 \times 2$ system. Apart from that, it is also found that the performance of the purposed model was also enhanced with more than $100 \%$ as compared to that of the $2 \times 2$ MIMO-VLC system with GRPA as reported by Chen et al. ${ }^{26}$ Overall, the enhancement varies from $138 \%$ at a low normalized offset up to $181 \%$ at a higher normalized offset. The challenge of using $4 \times 4$ MIMONOMA-VLC is that it will increase the complexity level of the system. This is because the system has to evaluate sixteen different vectors instead of only four in $2 \times 2$ MIMO-NOMAVLC system.

\subsubsection{Performance enhancement after applying GRPA with the $4 \times 4$ MIMO-NOMA at $K=2,3$, and 4}

Examining GRPA's effect on the $4 \times 4$ MIMO-NOMA-based VLC system, Fig. 3(b) and Table 3 shows the sum rate at $K=2,3$, and 4 before and after application of GRPA. It can be seen that the sum rate of the $4 \times 4$ MIMO-NOMA-based VLC system with GRPA for both $K=2,3$, and 4 was increased compared to that with OFDMA only. These findings clearly indicate the better performance for the device with GRPA.

In Fig. 3(b), the realizable sum rate versus $r / R$ for $4 \times 4$ MIMO-VLC systems with OFDMA and NOMA is demonstrated. As can be seen from the figure, the sum rate obtained using 
OFDMA is constantly decreasing with increasing $r / R$. For NOMA, the sum rate is constant over a broader range (i.e., $0.1 r / R=0.8$ ). For both $K=2$ and 3, a reduction in the total rate occurs only when $r / R>0.8$. This indicates that NOMA with GRPA only suffers losses in sum-rate when $r / R$ becomes relatively large. From Fig. 3(b), it can be deduced that the best system utilization is at $K=4$ because the sum rate is stable within the whole range of $0.1 \leq r / R \leq 0.9$, and the losses are $<1 \%$ of the maximum achievable sum rate. Therefore, the four users' case is the most utilized one because it enables the use of the entire bandwidth.

It is clear from Fig. 3(b) that after applying GRPA on $4 \times 4$ MIMO-NOMA-based VLC system, the system can utilize the maximum achievable sum rate for users regardless their current position. The maximum achievable sum rate is $268.55 \mathrm{Mbit} / \mathrm{s}$ at the center of the system for all users. At the edge of the system, the maximum achievable sum rates for 2,3 , and 4 users are 258.74, 264.64, and 266.20 Mbit/s, respectively. These sum rates are $96 \%, 98 \%$, and $99 \%$ as compared to that at the center of the system, respectively. In conclusion, $4 \times 4$ MIMO-NOMAbased system with GRPA scheme can maintain almost the same achievable sum rate for all users regardless of their current position.

\subsection{Sum-Rate Gain vs. Normalized Offset}

Figure 4 shows the GRPA sum-rate gain over OFDMA versus $r / R$. It can be clear seen that with a large normalized offset, the gain of the sum rate increases significantly. The percentage of the sum-rate gain for $K=4$ on the border of the system coverage is lower than that for $K=2$ and $K=3$ cases. This is due to user $K$ is situated within the system's edge coverage. Overall, the achievable sum-rate gain is $40.22 \%, 33.47 \%$, and $24.88 \%$ for $K=2,3$, and 4 , respectively. This means lower percentages of sum-rate gain for a larger number of users.

\section{Conclusions}

In this article, a $2 \times 2$ MIMO-NOMA-VLC is expanded to a $4 \times 4$ MIMO-NOMA-VLC. The results of the simulation show that the overall system sum rate was increased by up to $43 \%$. In addition, a low-complexity GRPA process, which uses the channel conditions of the users, has been proposed in a $4 \times 4$ MIMO-NOMA-VLC system for efficient power allocation. As compared to the OFDMA method, the sum rate of the proposed system was substantially improved to $40 \%, 33 \%$, and $24 \%$ for two, three, and four users, respectively. In addition enhancing the achievable sum rate, GRPA scheme maintains almost the same achievable sum rate for all users regardless of their position. Finally, the results implied that MIMO-NOMA is promising for potential high-speed multi-user VLC systems with the proposed GRPA approach.

\section{Acknowledgments}

This work was supported by Universiti Sains Malaysia through the GOT Incentive Grant (Account No. 1001/CINOR/822083).

\section{References}

1. J. M. Hamamreh, H. M. Furqan, and H. Arslan, "Classifications and applications of physical layer security techniques for confidentiality: a comprehensive survey," IEEE Commun. Surv. Tutorials 21(2), 1773-1828 (2019).

2. J. Shi et al., "Experimental demonstration of OQAM-OFDM based MIMO-NOMA over visible light communications," in Opt. Fiber Commun. Conf. Expo. OFC 2018-Proc.(c), OSA, pp. 1-3 (2018).

3. R. Mesleh and A. Al-Olaimat, "Acousto-optical modulators for free space optical wireless communication systems," J. Opt. Commun. Networking 10(5), 515-522 (2018).

4. M. S. M. Gismalla et al., "Improve uniformity for an indoor visible light communication system,” Int. J. Commun. Syst. 33(8), e4349 (2020). 
5. W. Liu and X. He, "Performance analysis of MIMO visible light based V2V communications," IEEE Veh. Technol. Conf., IEEE, pp. 1-4 (2019).

6. H. Yang et al., "Learning-based energy-efficient resource management by heterogeneous RF/VLC for ultra-reliable low-latency industrial IoT networks," IEEE Trans. Ind. Inf. 16(8), 5565-5576 (2020).

7. H. Le Minh et al., " $100-\mathrm{Mb} / \mathrm{s}$ NRZ visible light communications using a postequalized white LED," IEEE Photonics Technol. Lett. 21(15), 1063-1065 (2009).

8. D. Tsonev et al., "A 3-Gb/s single-LED OFDM-based wireless VLC link using a gallium nitride $\mu$ LED," IEEE Photonics Technol. Lett. 26(7), 637-640 (2014).

9. M. S. M. Gismalla et al., "Design of an optical attocells configuration for an indoor visible light communications system," AEU - Int. J. Electron. Commun. 112, 152946 (2019).

10. K. O. Akande, P. A. Haigh, and W. O. Popoola, "On the implementation of carrierless amplitude and phase modulation in visible light communication," IEEE Access 6(5), 60532-60546 (2018).

11. A. Khalid et al., "Design of MIMO-visible light communication transceiver using maximum rank distance codes," IEEE Access 7, 89128-89140 (2019).

12. A. H. Azhar, T. A. Tran, and D. O'Brien, "A gigabit/s indoor wireless transmission using MIMO-OFDM visible-light communications," IEEE Photonics Technol. Lett. 25(2), 171-174 (2013).

13. P. Wang, J. Xiao, and L. Ping, "Comparison of orthogonal and non-orthogonal approaches to future wireless cellular systems," IEEE Veh. Technol. Mag. 1(3), 4-11 (2006).

14. L. Dai et al., "Non-orthogonal multiple access for 5G: solutions, challenges, opportunities, and future research trends," IEEE Commun. Mag. 53(9), 74-81 (2015).

15. S. M. R. Islam et al., "Power-domain non-orthogonal multiple access (NOMA) in 5G systems: potentials and challenges," IEEE Commun. Surv. Tutorials 19(2), 721-742 (2017).

16. V. Ozduran, "Advanced successive interference cancellation for non-orthogonal multiple access," in 26th Telecommun. Forum, TELFOR 2018-Proc., IEEE, Vol. 2, No. (2), pp. 1-4 (2018).

17. C. J. Rapson et al., "Applying NOMA to undersampled optical camera communication for vehicular communication," in 11th Int. Conf. Mob. Comput. Ubiquitous Network, 2018, IPSJ (2019).

18. Z. Ding et al., "On the performance of non-orthogonal multiple access in $5 \mathrm{G}$ systems with randomly deployed users," IEEE Signal Process. Lett. 21(12), 1501-1505 (2014).

19. H. Yang et al., "Joint precoder and equalizer design for multi-user multi-cell MIMO VLC systems," IEEE Trans. Veh. Technol. 67(12), 11354-11364 (2018).

20. I. E. Lee, M. L. Sim, and F. W. L. Kung, "Performance enhancement of outdoor visible-light communication system using selective combining receiver," IET Optoelectron. 3(1), 30-39 (2009).

21. H. Marshoud et al., "On the performance of visible light communication systems with nonorthogonal multiple access," IEEE Trans. Wireless Commun. 16(10), 6350-6364 (2017).

22. X. Jing et al., "Dynamically connected hybrid precoding scheme for millimeter-wave massive MIMO systems," IEEE Commun. Lett. 22(12), 2583-2586 (2018).

23. Y. Liu et al., "Energy-efficient hybrid precoding with low complexity for mmWave massive MIMO systems," IEEE Access 7, 95021-95032 (2019).

24. Z. Ding, R. Schober, and H. V. Poor, "A general MIMO framework for NOMA downlink and uplink transmission based on signal alignment," IEEE Trans. Wireless Commun. 15(6), 4438-4454 (2016).

25. S. Tao et al., "Power allocation of non-orthogonal multiple access with variable on-off keying dimming control in visible light communication networks," in Int. Conf. Commun. Technol. Proc., ICCT, IEEE, pp. 321-325 (2019).

26. C. Chen et al., "On the performance of MIMO-NOMA-based visible light communication systems," IEEE Photonics Technol. Lett. 30(4), 307-310 (2018).

27. C. Chen, W. De Zhong, and D. Wu, "Non-Hermitian symmetry orthogonal frequency division multiplexing for multiple-input multiple-output visible light communications," J. Opt. Commun. Networking 9(1), 36-44 (2017). 
28. X. Liu et al., "Power allocation algorithm of optical MIMO NOMA visible light communications," in ICEIEC 2019-Proc. 2019 IEEE 9th Int. Conf. Electron. Inf. Emerg. Commun., pp. 504-508 (2019).

29. L. Zeng et al., "High data rate multiple input multiple output (MIMO) optical wireless communications using white LED lighting," IEEE J. Sel. Areas Commun. 27(9), 1654-1662 (2009).

30. C. Chen, W. De Zhong, and D. Wu, "Communication coverage improvement of indoor SDM-VLC system using NHS-OFDM with a modified imaging receiver," in IEEE Int. Conf. Commun. Work. ICC 2016, IEEE, pp. 315-320 (2016).

31. S. Tao et al., "Strategy-based gain ratio power allocation in non-orthogonal multiple access for indoor visible light communication networks," IEEE Access 7, 15250-15261 (2019).

32. Y. Saito et al., "System-level performance evaluation of downlink non-orthogonal multiple access (NOMA)," in IEEE Int. Symp. Pers. Indoor Mob. Radio Commun. PIMRC, IEEE, pp. 611-615 (2013).

Ibrahim A. Elewah received his MSc degree in 2015 from Arab Academy for Science, Technology \& Maritime Transport, Egypt. Currently, he is a PhD student at Universiti Sains Malaysia (USM), Malaysia. He is working at ACM in Kuwait as a team leader of the computer engineering technology program (ABET accredited). Ibrahim received two awards in 2019, the faculty award for outstanding teaching and learning, and the certificate of appreciation for contribution to ACM ABET accreditation. He is an IEEE senior member, member of OSA, and SPIE.

Faezah Jasman received her BEng (Hons) degree in electronics engineering from Multimedia University (MMU), MSc in communication network engineering from the University Putra Malaysia (UPM) and PhD from University of Warwick, UK. She is currently a senior lecturer at the Institute of Nano Optoelectronics Research and Technology (INOR), Universiti Sains Malaysia (USM). Her current research interests include modelling and characterization of underwater optical wireless channels and optoelectronic devices, modulation and coding schemes for VLC.

Shashiong Ng received his $\mathrm{PhD}$ from USM, Malaysia. At present, he is an associate professor at INOR, USM. His specialization is the growth and characterizations of wide band gap semiconductor materials. He has published more than 150 papers and his h-index is 18 . Currently, he is a senior member of OSA, and members of MRS and SPIE. 